\title{
Anti-stress Effects of Flavonoids from Buckwheat Sprouts in Mice Subjected to
}

\section{Restraint Stress}

\author{
Mitsuru Watanabe* and Jun AyUgase \\ National Agricultural Research Center for Tohoku Region, 4 Akahira, Shimokuriyagawa, Morioka, Iwate 020-0198, Japan
}

Received September 11, 2007; Accepted January 17, 2008

We examined the effects of orally administered flavonoids isolated from buckwheat (Fagopyrum esculentum Moench) sprouts in restrained mice. These flavonoids have been shown to have antioxidant activities in vitro. A propylene glycol (PPG) or flavonoid (mixture of rutin, orientin, isoorientin, vitexin, and isovitexin in PPG) solution (100 mg/kg body weight) was administered to mice intragastrically once per day for three days, after which the mice were restrained for $24 \mathrm{~h}$. Unrestrained mice were administered PPG solution with or without free access to feed and water. Restraint stress induced increases in plasma corticosterone, plasma glutamic-oxaloacetic transaminase activity, and the amount of thiobarbituric acidreactive substances in plasma and liver tissues. In contrast, these variables were suppressed in the mice that were administered flavonoids. These results suggest that flavonoids from buckwheat sprouts have in vivo anti-stress effects against the reactions induced by immobilization in mice.

Keywords: buckwheat sprout, flavonoid, antioxidant activity, restraint stress

\section{Introduction}

Many stressors cause physical and psychological stresses. Numerous studies using stress models have shown that physiological and psychological stress stimuli activate the sympathetic nervous system and hypothalamic-pituitaryadrenal (HPA) axis, which results in the secretion of catecholamines from the rat brain (Tanaka et al., 1983) and the adrenal medullary cells (Jung et al., 2000) and glucocorticoids from the adrenocortical cells (Takeuchi et al., 2001). Excess glucocorticoid concentrations can have harmful effects such as hypertension, ulcers, immunosuppression, and reproductive impairment (Sapolosky, 1994). In addition, it has been reported that restraint stress leads to an increase in reactive oxygen species (ROS) in the brain, plasma, and liver. Consequently, oxidants from stress could contribute to the degenerative diseases of aging, including brain dysfunction (Liu et al., 1996). Furthermore, an elevation of blood glucose, blood pressure, or lipids by stress stimuli results in the onset of lifestyle-related diseases such as diabetes (Stefan et al., 1971, Carter et al., 1987, Thernlund et al., 1995). Therefore, much attention has recently been focused

*To whom correspondence should be addressed.

Email:wata@affrc.go.jp on food components with anti-stress activities for preventing many diseases and morbid states. Buckwheat sprouts are rich sources of phytochemicals such as flavonoids (Watanabe and Ito, 2002) and anthocyanins with antioxidant activity (Watanabe, 2007). The protective effect of rutin, one of the major flavonoids in buckwheat sprouts, against gastric ulcer induced by $50 \%$ ethanol was ascribed to its antioxidant activity (La Casa et al., 2000). Cruz et al. (1998) reported that oral administration of rutin ameliorated inflammatory bowel disease induced by trinitrobenzenesulfonic acid in rats. Sai et al. (1998) showed that antioxidative green tea components were responsible for the effective inhibition of hepatic injuries induced by oral administration of 2-nitropropane, which generates ROS via its metabolites. Their results showed that antioxidative phenolic compounds can improve the dysfunction induced by oxidative stress. Moreover, some flavonoids inhibit the activity of xanthine oxidase, which catalyzes in vivo generation of superoxide anion radicals and uric acid. The inhibition of xanthine oxidase activity is expected to suppress the generation of superoxide anions and uric acid in cases of ischemia-reperfusion injury and gout, respectively (Nagao et al., 1999). Flavonoids and anthocyanins are common pigments widely distributed in the plant kingdom. Humans usually consume a large amount of flavonoids and 
anthocyanins from plant foods. In this study, we investigated the effects of orally administered flavonoids isolated from buckwheat sprouts in mice under restraint stress.

\section{Materials and Methods}

Flavonoids Etiolated buckwheat sprouts were grown as previously described (Watanabe and Ito, 2003). The harvested sprouts were freeze-dried and powdered using a vibrating sample mill. Flavonoids were extracted with methanol from the powdered sprouts under reflux in a water bath at $75^{\circ} \mathrm{C}$, and the extract was subjected to Sephadex LH-20 chromatography. The flavonoid fractions, obtained by elution with ethanol by monitoring absorbance at $350 \mathrm{~nm}$, were dried under reduced pressure, and the residue was dissolved in PPG at a concentration of $10 \mathrm{mg} / \mathrm{ml}$. The percentages (w/w) of flavonoid compounds (Fig. 1) in the solution, determined by HPLC analysis in the manner previously described (Watanabe and Ito, 2003), were as follows: rutin, 30\%; orientin, 12\%; isoorientin, 17\%; vitexin, 16\%; and isovitexin, 25\%.

Animals Female ddY mice were purchased from Japan SLC Inc. (Hamamatsu, Japan). They were housed in plastic cages in a temperature-controlled room $\left(23 \pm 1^{\circ} \mathrm{C}\right)$ under controlled light and dark conditions (12 h each), and had free access to water and feed (CE-2; Japan CLEA) until the experiment began. At eight weeks of age, the mice were randomly divided into four groups of 10 to 11 mice each. The mice in the restraint stress/flavonoid (RS) group were intragastrically administered $100 \mathrm{mg} / \mathrm{kg}$ body weight of buckwheat sprout flavonoid (BSF) solution $(10 \mathrm{mg} / \mathrm{ml}$ in PPG) three times at 24-h intervals and were subsequently restrained for $24 \mathrm{~h}$. The mice in the restraint stress/control (RC) group were intragastrically administered PPG solution and subsequently restrained for $24 \mathrm{~h}$. The mice in the no restraint
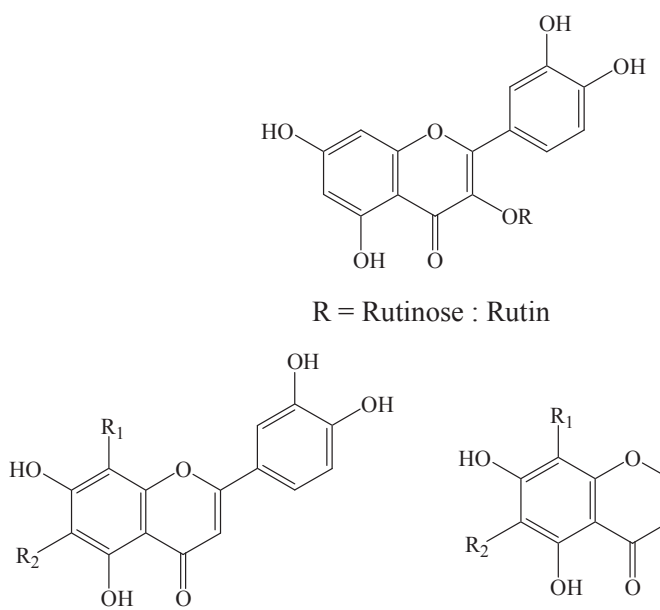

$\mathrm{R}_{1}=$ Glucose, $\mathrm{R}_{2}=\mathrm{H}:$ Orientin $\mathrm{R}_{1}=\mathrm{H}, \mathrm{R}_{2}=$ Glucose : Isoorientin

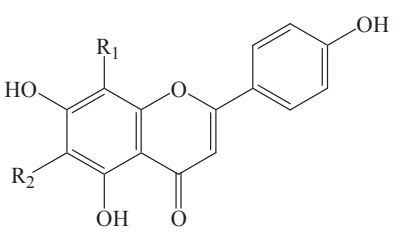

$\mathrm{R}_{1}=$ Glucose, $\mathrm{R}_{2}=\mathrm{H}:$ Vitexin $\mathrm{R}_{1}=\mathrm{H}, \mathrm{R}_{2}=$ Glucose $:$ Isovitexin

Fig. 1. Structures of flavonoid compounds in buckwheat sprouts. stress/fasting (NF) group were intragastrically administered PPG solution and subsequently fasted for $24 \mathrm{~h}$. The mice in the no restraint stress/control (NC) group were administered PPG solution with free access to water and feed. The mice received humane care as outlined in the Guide for the Care and Use of Experimental Animals (National Agricultural Research Center for Tohoku Region).

Stress protocol Thirty minutes after the last oral administration of BSF or PPG solution, the mice were subjected to restraint stress by using $50-\mathrm{ml}$ plastic tubes $(2.7 \mathrm{~cm} \times 10$ $\mathrm{cm}$ ) according to the method previously published (Bonneau et al., 1993, Iwakabe et al., 1998, and Kohno and Ohkubo, 1998). In brief, the mice were placed under restraint stress individually by placing each of them in a tube with holes $(0.4 \mathrm{~cm}$ diameter) for breathing for $24 \mathrm{~h}$. After being immobilized, the mice were killed by collecting whole blood from the heart in heparinized tubes, and the liver was excised. The plasma was prepared by centrifuging the collected blood at $10,580 \times \mathrm{g}$ for $5 \mathrm{~min}$ at $4^{\circ} \mathrm{C}$. The plasma and liver were stored at $-80^{\circ} \mathrm{C}$ until used.

Measurement of plasma corticosterone Plasma corticosterone, which is the main glucocorticoid in rodents, was extracted with ethyl acetate and measured by HPLC according to Hayashi's method (Hayashi et al., 2001). Cortisol was used as an internal standard.

Measurement of plasma glucose and insulin Plasma glucose was measured with a Glucose C-II Test Wako Kit (Wako Pure Chemical Ltd., Osaka, Japan) according to the instruction manual. Plasma insulin was assayed using an enzymelinked immunosorbent assay kit for mice (Shibayagi, Co., Ltd., Gunma, Japan).

Extraction of lipids from liver tissues Total lipids in liver tissues were extracted according to Folch's method with chloroform-methanol $(2: 1 ; \mathrm{v} / \mathrm{v})$ solvent (Folch et al., 1957). After removal of the solvent from the extracts, the residue was dissolved in 2-propanol containing 10\% Triton X-100. This crude lipid solution was used for the measurement of lipids.

Measurement of lipids Total cholesterol, HDL cholesterol, and triglyceride concentrations were measured with Cholesterol E Test, HDL Cholesterol E Test, and Triglyceride E Test kits, respectively (all from Wako Pure Chemical Ltd.).

Assay for plasma glutamic-oxaloacetic transaminase (GOT) and glutamic-pyruvic transaminase (GPT) Plasma GOT and GPT activities, enzyme markers for injury to the liver and other tissues, were assayed with a Transaminase C-II Test Wako kit (Wako Pure Chemical Ltd.) according to the instruction manual.

Assay for thiobarbituric acid reactive substances (TBARS) in liver and plasma The concentrations of TBARS in liver 
tissue homogenate were analyzed using Kikugawa's method (Kikugawa et al., 1992), which is a modification of Ohkawa' s method (Ohkawa et al., 1979). Plasma TBARS were analyzed according to Yagi's method (Yagi, 1976).

Measurement of protein content in the liver homogenate The protein concentration in the liver homogenate was measured by a Protein Quantification Kit-Rapid (Dojindo, Kumamoto, Japan).

Statistics Statistically significant differences between groups were evaluated by one-way analysis of variance (ANOVA) followed by the Tukey-Kramer test with the use of Prism 4 software (GraphPad Software Inc.). Differences at $P<0.05$ were considered to be significant.

\section{Results}

The effect of BSF on plasma corticosterone concentrations in restrained mice is shown in Fig. 2. The concentration of plasma corticosterone was higher in the $\mathrm{NF}$ and $\mathrm{RC}$ groups than in the $\mathrm{NC}$ group, and the concentration in the $\mathrm{RC}$ group tended to be higher than that in the NF group. The elevation of plasma corticosterone in mice in the NF and RC groups indicated that stressor stimuli-food restriction and immobilization - activated the HPA axis in these groups. In contrast, pretreatment of mice with BSF (RS group) significantly suppressed the elevation of plasma corticosterone compared with the RC group $(P<0.05)$.

The effect of BSF on plasma glucose concentrations in restrained mice is shown in Fig. 3. The plasma glucose concentration was lower in the $24 \mathrm{~h}$ of fasting (NF) and restraint ( $\mathrm{RC}$ and $\mathrm{RS}$ ) groups than in the NC group. Among the fasting and restraint groups, the plasma glucose concentration

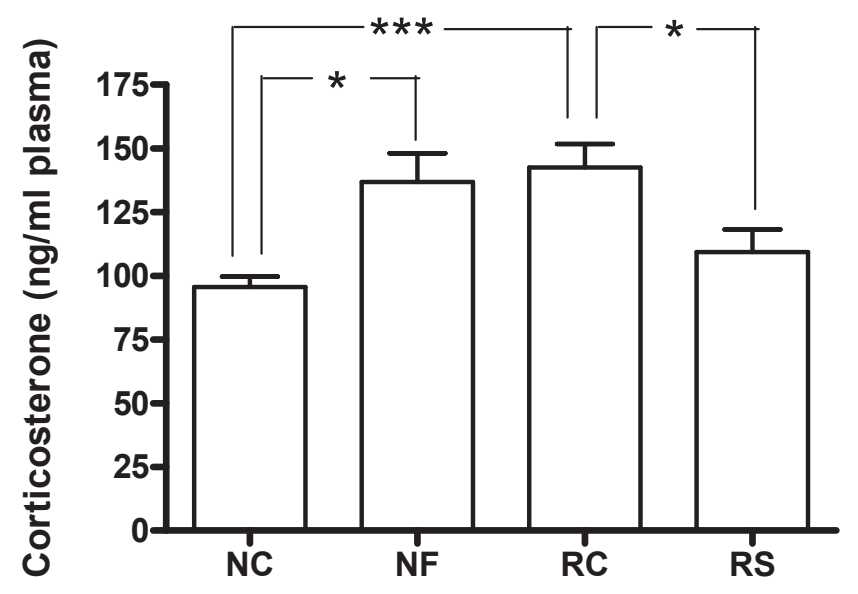

Fig. 2. Effect of buckwheat sprout flavonoids (BSF) on plasma corticosterone concentrations in restrained mice. $\mathrm{NC}$, no restraint stress/control group; NF, no restraint stress/fasting group; RC, restraint stress/control group; RS, restraint stress/flavonoid group. Data are mean $\pm \mathrm{SE}(\mathrm{n}=10 \sim 11)$. $P<0.05, * * * P<0.001$. was higher in the RC group than in the NF group, and administration of BSF suppressed glucose concentrations in the RS group compared with the RC group $(P<0.001)$. Although plasma insulin concentrations were lower in the RS group than in the NC group, concentrations were not significantly different between the NF, RC, and RS groups (Fig. 4).

Concentrations of total cholesterol, HDL cholesterol, and triglycerides and the arteriosclerotic index (total cholesterol - HDL cholesterol/HDL cholesterol) in plasma are shown in Table 1. Although the concentration of plasma total cholesterol was significantly higher in the NF, RC, and RS groups than in the $\mathrm{NC}$ group, it was lower in the RS group than in the RC group. In contrast, the concentration of plasma HDL

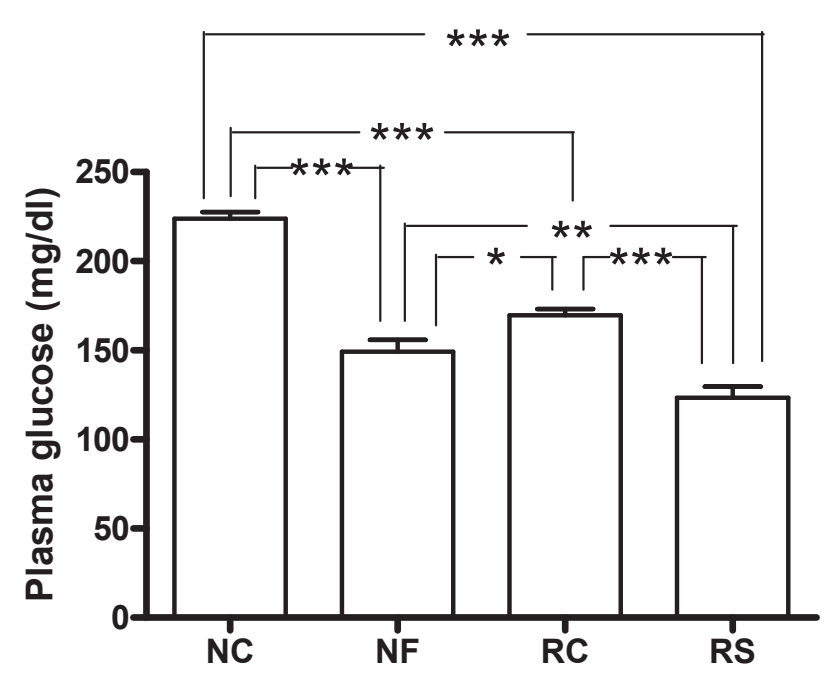

Fig. 3. Effect of buckwheat sprout flavonoids (BSF) on plasma glucose concentrations in restrained mice. NC, no restraint stress/ control group; NF, no restraint stress/fasting group; RC, restraint stress/control group; RS, restraint stress/flavonoid group. Data are mean $\pm \mathrm{SE}(\mathrm{n}=10)$. $* P<0.05, * * P<0.01$, *** $P<0.001$.

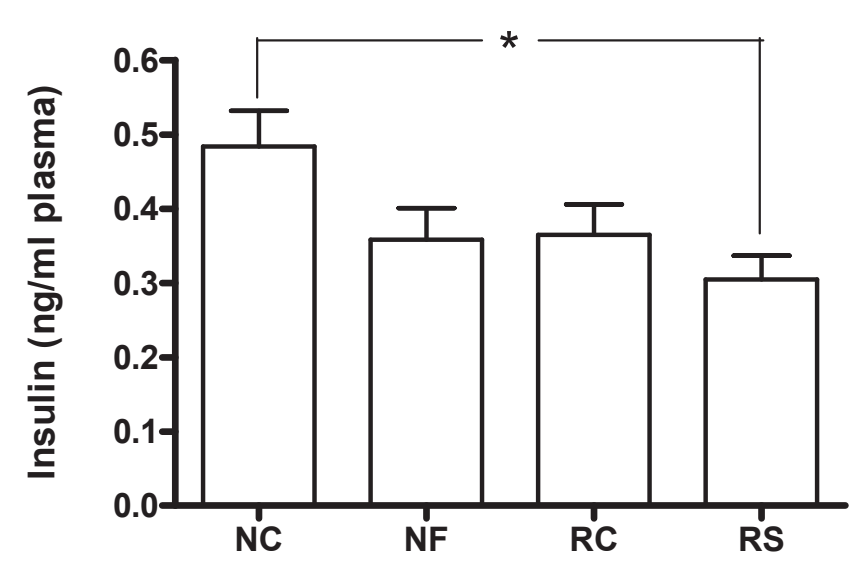

Fig. 4. Effect of buckwheat sprout flavonoids (BSF) on plasma insulin concentrations in restrained mice. NC, no restraint stress/ control group; NF, no restraint stress/fasting group; RC, restraint stress/control group; RS, restraint stress/flavonoid group. Data are mean $\pm \mathrm{SE}(\mathrm{n}=10)$. $* P<0.05$. 
Table 1. Effects of BSF on the plasma triglyceride concentrations, cholesterol concentrations, and arteriosclerotic index in restrained mice.

\begin{tabular}{crrrr}
\hline \hline Group & $\begin{array}{c}\text { Triglycerides } \\
(\mathrm{mg} / \mathrm{dl})\end{array}$ & $\begin{array}{c}\text { Total cholesterol } \\
(\mathrm{mg} / \mathrm{dl})\end{array}$ & $\begin{array}{c}\text { HDL cholesterol } \\
(\mathrm{mg} / \mathrm{dl})\end{array}$ & $\begin{array}{c}\text { Arteriosclerotic } \\
\text { index* }\end{array}$ \\
\hline $\mathrm{NC}(\mathrm{n}=10)$ & $117.2 \pm 11.6^{\mathrm{a}}$ & $91.1 \pm 1.6^{\mathrm{a}}$ & $59.5 \pm 0.7^{\mathrm{a}}$ & $0.53 \pm 0.04^{\mathrm{ab}}$ \\
$\mathrm{NF}(\mathrm{n}=11)$ & $52.0 \pm 2.9^{\mathrm{b}}$ & $107.8 \pm 1.8^{\mathrm{bc}}$ & $63.7 \pm 2.0^{\mathrm{ab}}$ & $0.71 \pm 0.06^{\mathrm{bc}}$ \\
$\mathrm{RC}(\mathrm{n}=10)$ & $48.0 \pm 2.2^{\mathrm{b}}$ & $115.5 \pm 3.9^{\mathrm{c}}$ & $66.6 \pm 3.0^{\mathrm{ab}}$ & $0.75 \pm 0.06^{\mathrm{c}}$ \\
$\mathrm{RS}(\mathrm{n}=11)$ & $45.4 \pm 2.6^{\mathrm{b}}$ & $102.4 \pm 2.5^{\mathrm{b}}$ & $71.2 \pm 1.8^{\mathrm{b}}$ & $0.44 \pm 0.03^{\mathrm{a}}$ \\
\hline
\end{tabular}

BSF, buckwheat sprout flavonoids; HDL, high-density lipoprotein; NC, no restraint stress/control group; $\mathrm{NF}$, no restraint stress/fasting group; RC, restraint stress/control group; RS, restraint stress/flavonoid group. Data are mean \pm SE. Different superscript letters indicate significant differences $(P<0.05)$.

*Arteriosclerotic index $=($ Total cholesterol - HDL cholesterol $) /$ HDL cholesterol

Table 2. Effects of BSF on hepatic lipid concentrations in restrained mice.

\begin{tabular}{ccc}
\hline \hline Group & $\begin{array}{c}\text { Triglycerides } \\
(\mathrm{mg} / \mathrm{g} \text { tissue })\end{array}$ & $\begin{array}{c}\text { Total cholesterol } \\
(\mathrm{mg} / \mathrm{g} \text { tissue })\end{array}$ \\
\hline $\mathrm{NC}(\mathrm{n}=10)$ & $6.6 \pm 2.0^{\mathrm{a}}$ & $2.5 \pm 0.1^{\mathrm{a}}$ \\
$\mathrm{NF}(\mathrm{n}=10)$ & $15.7 \pm 2.4^{\mathrm{b}}$ & $2.6 \pm 0.1^{\mathrm{ab}}$ \\
$\mathrm{RC}(\mathrm{n}=10)$ & $17.5 \pm 3.0^{\mathrm{b}}$ & $2.9 \pm 0.1^{\mathrm{ab}}$ \\
$\mathrm{RS}(\mathrm{n}=11)$ & $19.3 \pm 2.4^{\mathrm{b}}$ & $3.1 \pm 0.1^{\mathrm{b}}$ \\
\hline
\end{tabular}

$\mathrm{BSF}$, buckwheat sprout flavonoids; NC, no restraint stress/control group; $\mathrm{NF}$, no restraint stress/fasting group; $\mathrm{RC}$, restraint stress/control group; RS, restraint stress/flavonoid group. Data are mean \pm SE. Different superscript letters indicate significant differences $(P<0.05)$.

Table 3. Effects of BSF on plasma GOT and GPT activities in restrained mice.

\begin{tabular}{crr}
\hline \hline Group & $\begin{array}{c}\text { GOT activity } \\
(\mathrm{IU} / 1)\end{array}$ & $\begin{array}{c}\text { GPT activity } \\
(\mathrm{IU} / \mathrm{l})\end{array}$ \\
\hline NC $(\mathrm{n}=11)$ & $15.6 \pm 0.6^{\mathrm{a}}$ & $5.4 \pm 0.3$ \\
$\mathrm{NF}(\mathrm{n}=10)$ & $23.5 \pm 2.7^{\mathrm{b}}$ & $5.6 \pm 0.2$ \\
$\mathrm{RC}(\mathrm{n}=11)$ & $28.0 \pm 2.0^{\mathrm{b}}$ & $5.9 \pm 0.4$ \\
$\mathrm{RS}(\mathrm{n}=11)$ & $22.6 \pm 1.1^{\mathrm{b}}$ & $5.0 \pm 0.3$ \\
\hline
\end{tabular}

BSF, buckwheat sprout flavonoids; GOT, glutamic-oxaloacetictransaminase; GPT, glutamic-pyruvic transaminase; NC, no restraint stress/control group; NF, no restrait stress/fasting group; $\mathrm{RC}$, restraint stress/control group; RS, restraint stress/flavonoid group. Data are mean $\pm \mathrm{SE}$. Different superscript letters indicate significant differences $(P<0.05)$.

cholesterol was significantly higher in the RS group than in the NC group, so that the arteriosclerotic index in the RS group was substantially lower than that in the NF and RC groups $(P<0.05)$. On the other hand, the concentration of plasma triglycerides was significantly lower in the NF, RC, and $\mathrm{RS}$ groups than in the $\mathrm{NC}$ group, but it was not significantly different between the NF, RC, and RS groups. In liver tissues, the concentrations of triglycerides were higher in the $\mathrm{NF}, \mathrm{RC}$, and RS groups than in the NC group, but concentrations were not significantly different between the NF, RC, and RS groups. However, the concentration of total cholesterol was higher in the RS group than in the NC group (Table 2).

Plasma GOT and GPT activities - enzyme markers of injury to tissues such as the liver-are shown in Table 3. Although plasma GOT activity was significantly higher in the NF and RC groups than in the NC group, pretreatment with BSF tended to suppress the elevation in the activities of these enzymes compared with the RC group. In addition, the pattern of GPT activity among the groups was similar to that of GOT activity, i.e., GPT activity tended to be lower in the $\mathrm{RS}$ group than in the RC group. These results indicated that the damage to tissues induced by stress stimuli, including the liver, decreased with the administration of BSF in restrained mice.

TBARS concentrations in plasma and liver tissues are shown in Fig. 5. The data suggest that the stress of food restriction did not affect lipid peroxidation in vivo, whereas restraint stress elevated lipid peroxidation in both plasma and liver tissues. Pretreatment with BSF significantly suppressed the elevation of TBARS concentrations in both plasma and liver induced by restraint stress.

\section{Discussion}

The importance of identifying anti-stress components originating from natural sources is increasing, partly because oxidative stress, induced by physical and psychological stressors, leads to the insufficiency of organs such as the liver and induces morbid states, e.g., hyperglycemia and hypertension. Anti-stress effects of many food materials and 

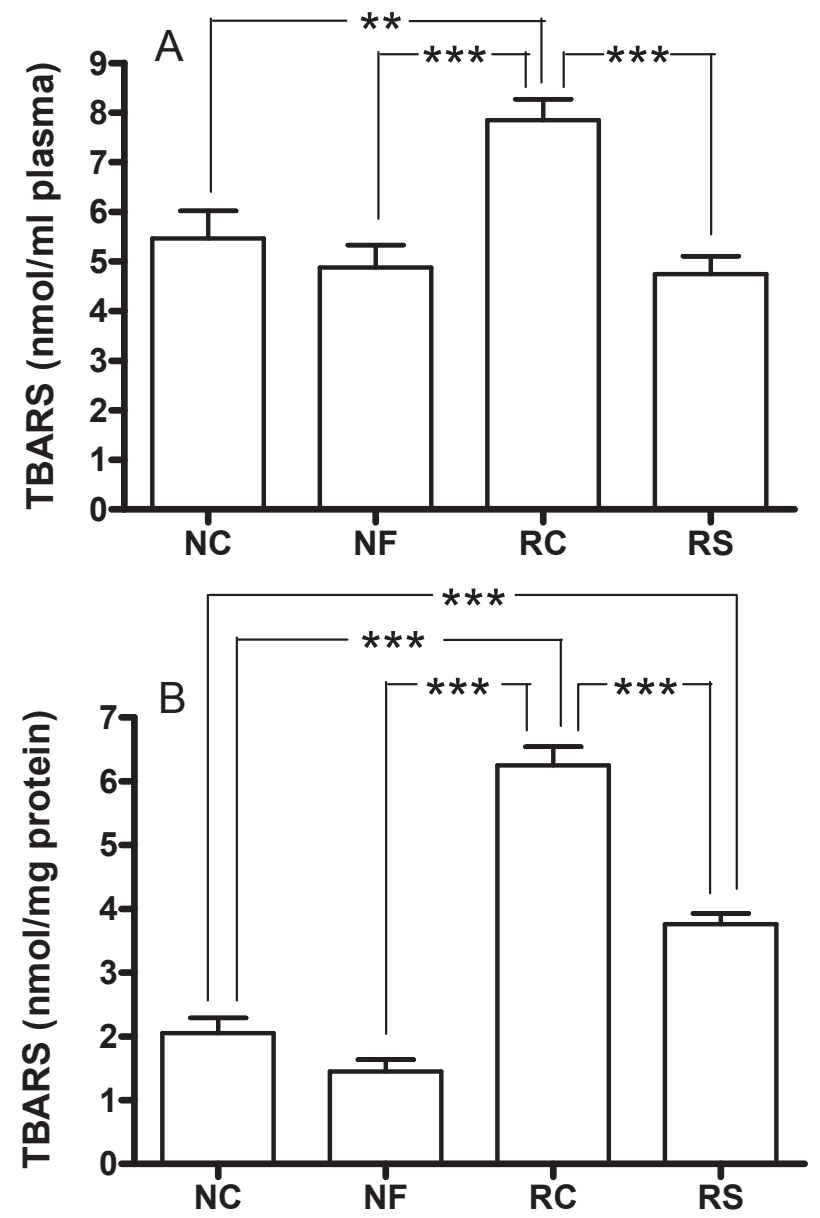

Fig. 5. Effects of buckwheat sprout flavonoids (BSF) on lipid peroxidation in plasma (A) and liver tissues (B) in restrained mice. $\mathrm{NC}$, no restraint stress/control group; NF, no restraint stress/fasting group; RC, restraint stress/control group; RS, restraint stress/flavonoid group. Data are mean $\pm \mathrm{SE}(\mathrm{n}=10 \sim 11)(\mathrm{A}) * * P<0.01, * * * P$ $<0.001$. (B) $* * * P<0.001$.

components have been investigated, and extracts from gamazumi (Iwai et al., 2001), oolong tea (Kurihara et al., 2004), and astaxanthin (Kurihara et al., 2002) have been reported to be effective in restrained rodents. Because the antioxidative activity of these food materials and components is extremely high, BSF, containing antioxidative flavonoid compounds, was expected to have anti-stress effects in restrained mice.

Although there have been numerous reports about the in vivo regulation of flavonoid compounds, most findings concerning their regulation focus on aglycones and $O$-glycosyl flavonoids. It was reported that rutin is hydrolyzed to its aglycone, quercetin, by the cecal microflora and is subsequently absorbed from the small intestine (Manach et al., 1997). In addition, the presence of aglycones and/or conjugates of some flavonoids such as naringenin (Peng et al., 1998), hesperetin (Tsai et al., 2000), epicatechin (Abd EI Mohsen et al., 2002), quercetin, and quercetin glycosides
(De Boer et al., 2005) in the brain after oral ingestion of flavonoids has been reported. The low distribution of quercetin in the brains of rats fed quercetin-containing diets (De Boer et al., 2005) suggests that BSF and/or its metabolites would be only slightly effective in the brain. Recently, we investigated the plasma concentration of each flavonoid compound in BSF $2 \mathrm{~h}$ after oral administration to mice and confirmed the presence of the intact form of $C$-glycosylflavones (data not shown) - the main components in BSF. This finding may be attributed to the difficulty with which cecal microflora hydrolyze $C$-glycosylflavonoids compared with $O$-glucosylflavonoids. However, clarification of the in vivo regulation of flavonoid compounds in BSF is needed.

The elevation of glucose concentrations in rodents subjected to restraint stress has been reported previously (Sugimoto et al., 1998, Kasuga et al., 1999), and the results of the earlier studies agree with the results of the present study. In emergency situations, the activation of the sympathetic nervous system by stressor stimuli induces the secretion of adrenaline from adrenal medullary cells. Subsequently, adrenaline suppresses the secretion of insulin from pancreatic $\beta$-cells, which results in an increase in the blood glucose concentration (Shimazu, 1981). DeTurck and Vogel (1980) have reported that values of plasma catecholamine levels reached a peak at 1-5 min and declined slowly thereafter toward baseline in immobilized rats. Kasuga et al. (1999) deduced that the effect of plasma catecholamine concentrations on insulin concentration was eliminated on exposure to restraint stress for $16 \mathrm{~h}$ per day for 2 consecutive days. Although plasma catecholamine concentrations were not determined in the present study, no significant difference in plasma insulin concentrations was noted between the RC and $\mathrm{RS}$ groups or the NC and RC groups. The results suggest that the effect of catecholamines on plasma glucose concentration via insulin at $24 \mathrm{~h}$ of fasting and immobilization would be minimal or eliminated.

Another factor affecting plasma insulin concentrations is cyclic adenosine monophosphate (cAMP), which regulates insulin secretion from the pancreas. An increase in the cAMP level induced by an agent could be interpreted as inhibition of cAMP-phosphodiesterase (i.e., the catabolic enzyme of cAMP) activity, which eventually, through stimulation of insulin secretion, reduces blood glucose concentration (Saltiel et al., 1986). Although it has been reported that the hypoglycemic effect of some flavonoids, such as rutin, vitexin and isovitexin, is attributable to the inhibition of cAMPphosphodiesterase, $C$-glycosides of luteolin, isoorientin and orientin were inactive (Petkov et al., 1981). However, Sezik et al. (2005) reported a hypoglycemic effect of isoorientin in Gentiana olivieri, and they also reported that the effect of 
isoorientin might not be dependent on cAMP-phosphodiesterase enzyme inhibition. Further evaluation of the effect of BSF on the insulin level is required.

After an emergency situation, chronic stress activates the HPA axis, resulting in elevation of plasma glucocorticoid concentrations from the adrenal cortex. Thereafter, elevation of the blood glucose concentration results from an increase in gluconeogenesis in the liver by glucocorticoids. In the present study, the plasma corticosterone concentrations were significantly higher in the NF and RC groups than in the NC group. These results suggested that the stress stimuli-fasting and immobilization - may have activated the HPA axis in mice. The suppression of the plasma corticosterone level in the RS group compared with the $\mathrm{RC}$ group raises the possibility that pretreatment with BSF may suppress activation of the HPA axis. Suppression of the blood glucose concentrations in mice administered BSF (RS group) compared with fasting (NF) and restraint stress/control (RC) groups could be attributed to the reduced plasma corticosterone concentration in addition to the hypoglycemic activity of flavonoid compounds in BSF including isoorientin. Thus, it is believed that BSF could prevent hyperglycemia induced by stress stimuli.

Regarding lipid metabolism, the plasma total cholesterol and HDL cholesterol concentrations were not significantly different between the RC and NF groups; therefore, restraint stress conditions in the present study would not be expected to affect the plasma cholesterol concentration. However, the plasma arteriosclerotic index improved in the RS group after pretreatment with BSF compared with the NF and RC groups. This effect was mainly attributed to suppression of the total cholesterol concentration, which was significantly lower in the RS group than in the RC group $(P<0.05)$, and to the increase in HDL cholesterol concentrations. Antihyperlipidemic activity, i.e., suppression of plasma cholesterol and triglycerides by rutin and isoorientin, has been reported in streptozotocin-induced diabetic rats (Stanley et al., 2006). The beneficial effect of rutin on lipid metabolism was attributed to its antioxidant activity. Therefore, the antihyperlipidemic effect of BSF in restrained mice could be ascribed to the flavonoid compounds with antioxidant activity in BSF, such as orientin, isoorientin, and rutin.

The brain is a target for different stressors because of its high sensitivity to stress-induced degenerative conditions (Sahin and Gumusul, 2004). It has been shown that restraint stress induced oxidative damage to the lipid, protein, and DNA in the brains of male Sprague-Dawley rats. Moreover, oxidative damage to the plasma and liver was similar to that in the brain (Liu et al., 1996). Because it is hypothesized that oxidants from stress could contribute to accelerated aging and degenerative diseases (Olanow, 1992), the inhibition of oxidative damage induced by stress stimuli should be expected. In the present study, although concentrations of TBARS in the plasma and liver of mice in the NF group were not significantly different from those in the $\mathrm{NC}$ group, concentrations of TBARS in the RC group were significantly higher than those in the $\mathrm{NC}$ group. In contrast, oral administration of BSF effectively suppressed the elevation of TBARS in plasma and liver, suggesting that the effects of BSF are attributable to the antioxidant activities of the flavonoid compounds in buckwheat sprouts against ROS induced by stress stimuli. In future studies, the effect of BSF on lipid peroxidation in the brain should be investigated along with its in vivo regulation.

In summary, the results of the present study confirm the anti-stress health benefits of BSF. In particular, BSF showed hypoglycemic and antihyperlipidemic effects and suppressed increased oxidative stress in vivo.

Acknowledgments This work was supported by a grant from the Ministry of Agriculture, Forestry and Fisheries of Japan for the project "Development of Evaluation and Management Methods for Supply of Safe, Reliable, and Functional Food and Farm Produce."

\section{References}

Abd El Mohsen, M.M., Kuhnle, G., Rechner, A.R., Schroeter, H., Rose, S., Jenner, P. and Rice-Evans, C.E. (2002). Uptake and metabolism of epicatechin and its access to the brain after oral ingestion. Free Radic. Biol. Med., 33, 1693-1702.

Bonneau, R.H., Sheridan, J.F., Feng, N. and Glaser, R. (1993). Stress-induced modulation of the primary cellular immune response to herpes simplex virus infection is mediated by both adrenal-dependent and independent mechanisms. J. Neuroimmunol., 42, 167-176.

Carter, W.R., Herrman, K.S., Stokes, K. and Cox, D.J. (1987). Promotion of diabetes onset by stress in the BB rat. Diabetologia, 30, 674-675.

Cruz, T., Galvez, J., Ocete, M.A., Crespo, M.E., Sanchez de Medina L.-H.F. and Zarzuedo, A. (1998). Oral administration of rutinoside can ameliorate inflammatory bowel disease in rats. Life Sci., 62, 687-695.

De Boer, V.C.J., Dihal, A.A., van der Woude, H., Arts, I.C.W., Wolffram, S., Alink, G.M., Retjens, I.M.C.M., Keijer, J. and Hollman, P.C.H. (2005). Tissue distribution of quercetin in rats and pigs. J. Nutr., 135, 1718-1725.

DeTurck, K.H. and Vogel, W.H. (1980).Factors influencing plasma catecholamine levels in rats during immobilization. Pharm. Biochem. Behav., 13, 129-131.

Folch, J., Lees, M. and Stanley, G.H.S. (1957). A simple method for isolation and purification of total lipid from animal tissues. $J$. Biol. Chem., 226, 497-509. 
Hayashi, T., Kataoka, H., Semma, M. and Ito, Y. (2001). Effects of stress on allergic reaction. Yakugaku Zasshi, 121, 753-759.

Iwakabe, K., Shimada, M., Ohta, A., Yahata, T., Ohmi, Y., Habu, S. and Nhishimura, T. (1998). The restraint stress drives a shift in Th1/Th2 balance toward Th2-dominant immunity in mice. Immunol. Lett., 62, 39-43.

Iwai, K., Onodera, A. and Matsue, H. (2001). Antioxidant activity and inhibitory effect of Gamazumi (Viburnum dilatatum THUNB.) on oxidative damage induced by water immersion restraint stress in rats. Int. J. Food Sci. Nutr., 52, 443-451.

Jung, B.D., Kimura, K., Kitamura, H., Makondo, K., Kanehira, K. and Saito, M. (2000). Sympathetic activation of hepatic and splenic IL-1 $\beta$ mRNA expression during oscillation stress in the rat. J. Vet. Med. Sci., 62, 409-413.

Kasuga, S., Ushijima M., Morihara, N., Itakura, Y. and Nakata, Y. (1999). Effect of aged garlic extract (AGE) on hyperglycemia induced by immobilization stress in mice. Folia Pharmacol. Jpn. (in Japanese), 114, 191-197.

Kikugawa, K., Kojima, T., Yamaki, S. and Kosugi, H. (1992). Interpretation of the thiobarbituric acid reactivity of rat liver and brain homogenates in the presence of ferric ion and ethylenediaminetetraacetic acid. Anal. Biochem., 202, 249-255.

Kohno, H. and Ohkubo, Y. (1998). Comparative glucoregulatory responses of mice to restraint and footshock stress stimuli. Biol. Pharm. Bull., 21, 113-116.

Kurihara, H., Koda, H., Asami, S., Kiso, Y. and Tanaka, T. (2002). Contribution of the antioxidative property of astaxanthin to its protective effect on the promotion of cancer metastasis in mice treated with restraint stress. Life Sci., 70, 2509-2520.

Kurihara, H., Fukami, H., Asami, S., Toyoda, Y., Nakai, M., Shibata, H. and Yao, X.S. (2004). Effects of oolong tea on plasma antioxidative capacity in mice loaded with restraint stress assessed using the oxygen radical absorbance capacity (ORAC) assay. Biol. Pharm. Bull., 27, 1093-1098.

La Casa, C., Villegas, I., Alarcon de la Lastra, C., Motiva, V. and Martin, M.J. (2000). Evidence for protective and antioxidant properties of rutin, a natural flavone, against ethanol induced gastric lesions. J. Ethnopharmacol., 71, 45-53.

Liu, J., Wang, X., Shigenaga, M.K., Yeo, H.C., Mori, A. and Ames, B.N. (1996). Immobilization stress causes oxidative damage to lipid, protein, and DNA in the brain of rats. FASEB J., 10, 1532-1538.

Manach, C., Morand C., Demigne, C., Texier, O., Regerat, F. and Remesy, C. (1997). Bioavailability of rutin and quercetin in rats. FEBS Lett., 409, 12-16.

Nagao, A., Seki, H. and Kobayashi, H. (1999). Inhibition of xanthine oxidase by flavonoids. Biosci. Biotechnol. Biochem., 63, 1787-1790.

Ohkawa, H., Ohishi, N. and Yagi, K. (1979). Assay for lipid peroxides in animal tissues by thiobarbituric acid reaction. Anal. Bio- chem., 95, 351-358.

Olanow, C.W. (1992). An introduction to the free radical hypothesis in Parkinson's disease. Ann. Neurol., 32, S2-S9.

Peng, H.W., Cheng, F.C., Huang, Y.T., Chen, C.F. and Tsai, T.H. (1998). Determination of naringenin and its glucuronide conjugate in rat plasma and brain tissue by high-performance liquid chromatography. J. Chromatogr. B, 714, 369-374.

Petkov, E., Nikilov, N. and Uzunov, P. (1981). Inhibitory effect of some flavonoids and flavonoid mixtures on cyclic AMP phosphodiesterase activity of rat heart. Planta Med., 43, 183-186.

Sahin, E. and Gumusul, S. (2004). Alterations in brain antioxidant status, protein oxidation and lipid peroxidation in response to different stress models. Behav. Brain Res., 155, 241-248.

Sai, K., Kai, S., Umemura, T., Tanimura, A., Hasegawa, R., Inoue, T. and Kurokawa, Y. (1998). Protective effects of green tea on hepatotoxicity, oxidative DNA damage and cell proliferation in the rat liver induced by repeated oral administration of 2-nitropropane. Food Chem. Toxicol., 36, 1043-1051.

Saltiel, A.R., Fox, J.A., Sherline, P. and Cuatrecasas, P. (1986) Insulin-stimulated hydrolysis of a novel glycolipid generates modulators of cAMP phosphodiesterase. Science, 233, 967-972.

Sapolsky, R.M. (1994). Glucocorticoids, stress and exacerbation of excitotoxic neuron death. Sem. Neurosci., 6, 323-331.

Sezik, E., Aslan, M., Yesilada, E. and Ito, S. (2005). Hypoglycaemic activity of Gentiana olivieri and isolation of the active constituent through bioassay- directed fractionation techniques. Life Sci., 76, 1223-1238.

Shimazu, T. (1981). Central nervous system regulation of liver and adipose tissue metabolism. Diabetologia, 20, 343-356.

Stanely Mainzen Prince, P. and Kannan, N.K. (2006). Protective effect of rutin on lipids, lipoproteins, lipid metabolizing enzymes and glycoproteins in streptozotocin-induced diabetic rats. $J$. Pharm. Pharmacol., 58, 1373-1383.

Stefan, P., Stein, M.D. and Edward, C.M.A. (1971). Emotional factors in juvenile diabetes mellitus: a study of early life experience of adolescent diabetics. Am. J. Psychiatr., 128, 700-704.

Sugimoto, Y., Yamada, J. and Noma, T. (1998). Effects of anxiolytics diazepam and tandospirone on immobilization stress-induced hyperglycemia in mice. Life Sci., 63, 1221-1226.

Takeuchi, H., Suzuki, N., Tada, M. and He, P. (2001). Accelerative effect of olive oil on liver glycogen synthesis in rats subjected to water-immersion restraint stress. Biosci. Biotechnol. Biochem., 65, 1489-1494.

Tanaka, M., Kohno, Y., Nakagawa, R., Ida, Y., Takeda, S., Nagasaki, N. and Noda, Y. (1983). Regional characteristics of stressinduced increases in brain noradrenaline release in rats. Pharm. Biochem. Behav., 19, 543-547.

Thernlund, G.M., Dahlquist, G., Hansson, K. and Ivarsson, S.A. (1995). Psychological stress and the onset of IDDM in children. Diabetes Care, 18, 1323-1329. 
Tsai, T.-H. and Chen, Y.-F. (2000). Determination of unbound hesperetin in rat blood and brain by microdialysis coupled to microbore liquid chromatography. J. Food Drug Anal., 8, 331-336.

Watanabe, M. (2007). An anthocyanin compound in buckwheat sprouts and its contribution to antioxidant capacity. Biosci. Biotechnol. Biochem., 71, 579-582.

Watanabe, M. and Ito, M. (2002). Changes in antioxidative activity and flavonoid composition of extracts from aerial parts of buck- wheat during the growth period. J. Jpn. Soc. Food Sci. Technol. (in Japanese), 49, 119-125.

Watanabe, M. and Ito, M. (2003). Effect of light on the content of phenolic compounds in buckwheat seedlings. J. Jpn. Soc. Food Sci. Technol. (in Japanese), 50, 32-34.

Yagi, K. (1976). A simple fluorometric assay for lipoperoxide in blood plasma. Biochem. Med., 15, 212-216. 